\title{
Normas en la formación didáctico-matemática de futuros profesores de primaria
}

Standards in the didactic-mathematical training of future primary teachers

Artículo de investigación| Research article

Fecha de recepción: 18 de febrero de 2019 Fecha de aceptación: 19 de julio de 2019 Fecha de disponibilidad en línea: diciembre de 2020

doi: 10.11144/Javeriana.m13.nfdm

Rocío Toscano rtoscano@us.es Universidad de SeVILLA, España ORCID: https://orcid.org/0000-0003-3396-268X

José María GaVILÁN-IZQUIERdo gavilan@us.es Universidad de SeVILLA, España D ORCID: https://orcid.org/0000-0002-3369-5377

Victoria SÁnchez vsanchez@us.es

Universidad de SeVILLA, España ORCID: https://orcid.org/0000-0003-2134-8025

Para citar este artículo | To cite this article Toscano, R., Gavilán-Izquierdo, J. M. \& Sánchez, V. (2020). Normas en la formación didáctico-matemática de futuros profesores de primaria. magis, Revista Internacional de Investigación en Educación, 13, 1-22. doi: 10.11144/ Javeriana.m13.nfdm 


\title{
Resumen
}

Este estudio se centra en la identificación de normas en el discurso coloquial que surge entre estudiantes para ser profesores (EP) de primaria cuando resuelven en grupo tareas didáctico-matemáticas. Los datos son las narrativas identificadas en las transcripciones de las grabaciones en audio de los diálogos de estos estudiantes. El análisis realizado ha permitido inferir la existencia de diferentes normas: sociales, sociomatemáticas, sociodidáctico-matemáticas y didácticomatemáticas. Su identificación ha permitido aproximarnos a cómo estas normas regulan la enseñanza/aprendizaje de estos estudiantes y pueden afectar su futuro desarrollo profesional como profesores de primaria.

\section{Palabras clave}

Formación de profesores de primaria; normas; trabajos prácticos

\section{Abstract}

This study focuses on the identification of standards in the everyday discourse that arises among student teachers of primary education when they resolve a set of didacticmathematical tasks. From the adopted commognitive framework, we consider the narrative. The data is the identified narratives in the transcriptions of the recorded dialogues of these students. The analysis has made it possible to infer the existence of different standards: social, sociomathematical and dicatic-mathematical. Their identification has allowed us to get closer to how these standards regulate the teaching/learning of these students and the fact that they can affect their future professional development as primary school teachers.
\end{abstract}

\section{Keywords}

Primary teacher education; norms; practical work (learning method) 
Descripción del artículo | Article description

Este artículo de investigación presenta un estudio empírico

de carácter cualitativo que hace parte del proyecto de

tesis doctoral titulado Discurso, normas e identidades en

estudiantes para maestros en relación con las matemáticas.

\section{Introducción}

En los últimos años la investigación educativa desde una perspectiva cognitiva se ha visto ampliada con la emergencia de diferentes perspectivas teóricas agrupadas bajo la denominación de perspectivas socioculturales, que también han sido descritas con denominaciones como sociohistóricas o histórico-culturales (Lave \& Wenger, 1991; Wells \& Claxton, 2002; Wertsch, 1985). En mayor o menor medida las aproximaciones socioculturales tienen un origen común en los trabajos de Vygotsky (1978), compartiendo la idea de que "comunicación, pensamiento y aprendizaje son procesos relacionados que están modelados por la cultura" (Mercer, 2004, p. 138). Según este último autor, los sucesos comunicativos se modelan por factores históricos y culturales, y el pensamiento y el aprendizaje no se pueden entender sin tener en cuenta la naturaleza social y comunicativa de la vida humana. En este contexto, el discurso que modela la interacción interpersonal se vuelve un elemento clave. Es importante destacar que en lo que coinciden diferentes investigadores es en que el discurso depende de la comunidad social en la que ocurre, tiene varios significados e implica el lenguaje, representaciones y comportamientos incrustados en la práctica (Gee, 1996; Moschkovich, 2007). Así, existen diferentes discursos asociados a colectivos, como médicos, juristas, deportistas, etc. (Gee, 1996; 2001a; 2001b).

El discurso matemático es un caso específico de comunicación, en el cual se incluyen números, formas geométricas, operaciones, etc., el cual se puede dar con diferentes matices en diferentes colectivos (profesores de matemáticas, alumnos de matemáticas, matemáticos, etc.). En las últimas décadas, ese discurso ha sido el foco de investigación de autores como, por ejemplo, Hunter \& Anthony (2011), que han estudiado la emergencia de un discurso matemático específico en las clases escolares, o Caspi \& Sfard (2012), que han considerado el progreso de los estudiantes en las clases de álgebra desde la charla algebraica informal hasta el discurso algebraico formal. Asumida la existencia de diferentes discursos, coincidimos con Sfard (2001) en que "el discurso matemático se hace especial por dos factores 
principales: primero, por su excepcional dependencia de artefactos simbólicos como sus herramientas de mediación de comunicación, y segundo, por las meta-normas particulares que regulan este tipo de comunicación" (p. 13). Partimos de esta idea y la extendemos a un discurso didáctico-matemático, que se supone que es el que se pretende generar en las aulas de los programas de formación de profesores en relación con las matemáticas, contexto en el que se sitúa este trabajo. Consideramos que, en este discurso, el uso que se hace de los artefactos simbólicos en las situaciones de enseñanza/aprendizaje y las metanormas particulares que esas situaciones generan son en este caso los factores importantes.

Desde una perspectiva sociológica, estas normas o prescripciones han sido especificadas como demandas, si se muestran de manera obvia, o normas, si son supuestos ocultos o ideas subyacentes (Biddle \& Thomas, 1996). Autores como Herbel-Eisenmann (2003) han proporcionado información sobre diferentes terminologías que han sido utilizadas con respecto al uso de la palabra norma, según la perspectiva adoptada.

En cuanto a la identificación de normas, son numerosos los trabajos que han tratado de caracterizar las distintas normas en diferentes contextos. Así, Yackel \& Cobb (1996) utilizan la noción de las normas sociomatemáticas, consideradas como "los aspectos normativos de discusiones matemáticas específicos de la actividad matemática de los estudiantes" (p. 461). Otros autores han usado las normas sociomatemáticas con un mayor peso social que en la interpretación de Yackel \& Cobb, enfocándose en las distintas reinterpretaciones de las mismas normas en las aulas multiétnicas, donde los estudiantes inmigrantes sienten que ellos mismos o sus contribuciones eran valoradas negativamente (Gorgorió \& Planas, 2005).

En un contexto de formación de profesores, Tatsis \& Koleza (2008) se han centrado en normas sociales y sociomatemáticas, estudiando cómo se establecen durante las interacciones de futuros profesores de primaria cuando resuelven problemas matemáticos, y su efecto en el proceso de resolución de problemas. En este mismo contexto, Sánchez \& García (2014) se han ocupado de las normas sociomatemáticas y matemáticas que surgen en la interacción entre estudiantes para profesores (EP) de primaria cuando resuelven una tarea matemática, incorporando el tema matemático tratado (la definición, en su caso) como una nueva variable. Con base en las aportaciones de Sfard (2008), Sánchez \& García (2014) asumen que las normas sociomatemáticas provienen de la manera de considerar las matemáticas como una materia escolar en un contexto específico. En Toscano, Sánchez \& García (2013), y en el caso de formación de profesores de secundaria, se ha estudiado la relación entre las normas socio-didáctico-matemáticas y la futura perspectiva profesional que puedan adoptar como profesores. Para 
ellas, las normas socio-didáctico-matemáticas vienen de la manera de considerar la enseñanza y el aprendizaje de las matemáticas como una materia en un contexto específico: la formación de profesores.

El estudio que aquí presentamos es parte de una investigación más amplia, la cual tiene tres ejes clave: el discurso, las normas y la idea de identidad. La importancia alcanzada por estos aspectos, su relación con la manera de abordar la práctica docente y la posible influencia que pueden tener en el proceso de formación de los EP de primaria es el marco en el que se sitúa nuestra problemática de investigación. Un primer resultado de esta investigación lo constituyó la identificación en el discurso coloquial, mantenido por los EP de primaria cuando resuelven tareas didáctico-matemáticas planteadas en la parte práctica de un curso de Didáctica de las Matemáticas, de la coexistencia de dos tipos de discursos: uno, el generado por los EP cuando adoptan el papel de alumnos que tienen que resolver una tarea planteada en el aula por su profesor (universitario, en este caso), con características que provienen de las experiencias previas de los alumnos en las aulas escolares, en general, y de matemáticas, en particular; el otro, ligado a la adopción en la resolución de un papel vinculado a su futura labor profesional, relacionado con aspectos de la manera de considerar las matemáticas como una materia escolar que tiene que ser enseñada y aprendida en un contexto escolar (Toscano, Gavilán-Izquierdo \& Sánchez, 2019). Todo ello nos lleva a situar nuestro problema de investigación en plantearnos si esos discursos pueden estar regulados de algún modo por la existencia de determinadas normas, con lo que aquí abordaríamos el segundo eje de la investigación global.

\section{Marco teórico}

Dada la problemática en la que se sitúa la investigación que estamos desarrollando, adoptamos una perspectiva sociocultural, y dentro de ella tomamos como referencia el marco comognitivo, propuesto por Sfard (2008). Esta autora sugirió el término commognition para señalar la unidad de la comunicación y el pensamiento (cognición). Para Sfard (2006; 2008), pensar es una forma de comunicación interpersonal (comunicación consigo mismo). Ella considera el discurso como un "tipo especial de comunicación" (Sfard, 2008, p. 297), y lo convierte en el objeto de estudio. Sfard (2007; 2008) considera cuatro propiedades para caracterizar el discurso: uso de palabras, narrativas, mediadores visuales y rutinas. Como ya hemos mencionado anteriormente, consideramos un discurso didáctico-matemático relacionado con la triada alumnos-profesor-contenido, en el cual se incluyen expresiones relacionadas con la enseñanza y el aprendizaje de las 
matemáticas, y se adaptan a este discurso las propiedades propuestas por Sfard, adaptación ya validada en anteriores estudios (Toscano et al., 2019).

Dado el papel relevante que desempeñan las narrativas en la identificación de las normas, objeto de este estudio, en él asumimos que las narrativas son "una serie de expresiones, habladas o escritas, que se enmarcan como una descripción de objetos, de relaciones entre objetos, o procesos con o por objetos, y que están sujetas a aprobación o rechazo, es decir, a ser etiquetadas como 'verdaderas' o 'falsas'" (Sfard, 2008, p. 300), estando estos objetos relacionados con la triada alumnos-profesor-contenido.

En la teoría de la comognición, el aprendizaje es considerado como un cambio en el discurso, que se estimula a través del conflicto comognitivo, es decir, cuando los diferentes interlocutores que participan en el discurso intervienen conforme a distintas reglas discursivas (Sfard, 2007).

Para esta autora, existen unas normas que se consideran como "reglas metadiscursivas que son ampliamente endosadas y promulgadas dentro del discurso de la comunidad" (Sfard, 2008, p. 300). Compartimos en este trabajo este significado $y$, sin minimizar la importancia de otras normas, asumimos la existencia de unas normas sociales y sociomatemáticas que provienen de las experiencias previas de los alumnos en las aulas escolares, en las que, en diferentes contextos, niveles y con diferentes profesores, han cursado diferentes asignaturas, en general, y la asignatura de Matemáticas, en particular. Aquí nos centramos tanto en esas normas como en las normas socio-didáctico-matemáticas o didáctico-matemáticas que pueden surgir en la interacción entre EP de primaria cuando resuelven tareas relacionadas con la actividad profesional específica del profesor, como pueden ser el análisis de actividades escolares o la interpretación de las respuestas de los alumnos. En particular, en este estudio, se considera que

- las normas sociales son aquellas que se han establecido conjuntamente por un profesor y unos estudiantes como miembros de una misma comunidad generada en el aula (actual o pasada), donde dichas normas se definen (o se han definido) configurando los roles del profesor y los estudiantes.

- Las normas sociomatemáticas provienen de la manera de considerar las matemáticas como una materia escolar en unos contextos específicos (Sánchez \& García, 2011; 2014), situados en nuestro caso en el pasado de estos estudiantes universitarios (EP) como aprendices de matemáticas.

- Las normas socio-didáctico-matemáticas vienen de la manera de considerar la enseñanza y el aprendizaje de las matemáticas (didáctica de las matemáticas) por parte de los estudiantes universitarios 
(EP) en un contexto específico: la formación de profesores (Toscano et al., 2013). Estas normas pueden ser normas previas (que los EP traen al aula universitaria) sobre las relaciones entre el papel de un profesor, en general, unos alumnos y un contenido, que en este caso se concreta en un contenido didáctico-matemático, o ser generadas con el propio contenido didáctico-matemático.

- Las normas didáctico-matemáticas comprenden los requerimientos, las reglas o los convenios de carácter normativo relacionados con la didáctica de las matemáticas, establecidos en esa comunidad científica y aceptados comúnmente dentro de ella.

En este estudio no se consideran las normas que se pueden establecer en la interacción entre el profesor universitario y sus estudiantes, sino que nos centramos en aquellas normas que se identifican en la interacción entre los propios EP. Además de las normas anteriormente mencionadas, se asume que pueden existir otras normas de muy distinto tipo, pero no se abordan en este estudio. En él pretendemos indagar sobre qué tipo de normas subyacen en los discursos de los EP de primaria cuando resuelven tareas didáctico-matemáticas, y nos planteamos como objetivos identificarlas y aproximarnos a sus características y al papel que pueden cumplir.

\section{Método}

Teniendo en cuenta el paradigma sociocultural en el que se sitúan nuestros objetivos de investigación, este estudio adopta un enfoque cualitativo e interpretativo, tratando de describir, decodificar e interpretar el significado de eventos que suceden en un contexto social particular.

\section{Sujetos y contexto}

Los participantes en la investigación global fueron 88 EP de primaria, de los cuales 66 eran mujeres y 22 hombres. Estos EP participaron voluntariamente en ella, por lo que el criterio de selección fue su total disponibilidad para colaborar. Concretamente, el estudio aquí presentado es una parte de la investigación global, que se centra en 45 de los 88 EP de primaria participantes, de los cuales 38 eran mujeres y 7 hombres. Estos estudiantes estaban distribuidos en 11 subgrupos (nombrados en este estudio como SG1, SG2, etc.). La reducción de la muestra se debió a que estos EP compartían un mismo profesor universitario, con lo que, dados nuestros objetivos, se minimizaba la influencia que pudiese tener el que tuviesen distintos profesores. Todos ellos estaban matriculados en el Grado de Educación Primaria, concretamente en $2 .^{\circ}$ curso, y cursaban la asignatura Didáctica de las Matemáticas. 
Esta asignatura está compuesta por clases de teoría (2 horas semanales) y clases de prácticas (1 hora semanal, en la que se desglosa el grupo de teoría en dos grupos de práctica), siendo en estas últimas donde se llevó a cabo la investigación. En estas clases prácticas, los EP trabajan en pequeños grupos, compuestos de 3 a 6 estudiantes, y resuelven tareas didácticomatemáticas planteadas por los profesores universitarios de la asignatura.

Las tareas didáctico-matemáticas son comunes a todos los grupos de la asignatura. Estas tareas son muy diferentes a las tareas matemáticas a las que, hasta el momento, se han enfrentado estos estudiantes. Específicamente, presentan diferentes situaciones relacionadas con actividades profesionales que estos EP tienen que desarrollar después como profesores y diferentes contenidos matemáticos (Sánchez \& García, 2009). Por ejemplo, una tarea didáctico-matemática puede estar relacionada con actividades profesionales como analizar o diseñar actividades escolares, interpretar las respuestas de los alumnos, etc., en relación con contenidos como números, geometría, etc. Algunas herramientas conceptuales (como artículos en revistas, entre otras) se proporcionan para permitir resolver la tarea, y los grupos tienen una autonomía total con respecto a su forma de trabajar.

El estudio aquí presentado se realizó cuando los EP resolvían dos tareas didáctico-matemáticas planteadas en la parte práctica de la asignatura.

La primera tarea correspondía al contenido de geometría y a las actividades profesionales de analizar actividades escolares e interpretar las respuestas de los alumnos. En la tarea didáctico-matemática se incluía una actividad escolar en la que se representaban diferentes cuadriláteros y una serie de preguntas relacionadas con la identificación y clasificación de dichos cuadriláteros (basada en Burger \& Shaughnessy, 1986). A los EP se les demandaba, en primer lugar, analizar dicha actividad escolar en relación con los procesos de razonamiento geométrico y las estrategias que se podrían desarrollar. En segundo lugar, se les pedía plantear la actividad a alumnos de educación primaria (de 6 a 12 años) para, posteriormente, interpretar sus respuestas, teniendo en cuenta los niveles de razonamiento de Van Hiele. Para resolver esta tarea didáctico-matemática, las herramientas conceptuales procedían de la información proporcionada en la parte teórica de la asignatura y de algunos artículos proporcionados en la parte práctica (Gutiérrez \& Jaime, 1998; Hoffer, 1981; Jaime \& Gutiérrez, 1990).

La segunda tarea correspondía al contenido de probabilidad y a las actividades profesionales de analizar actividades escolares y formular preguntas para los alumnos. En la tarea didáctico-matemática se incluía una colección de problemas escolares relacionados con la probabilidad, extraídos de libros escolares y páginas web. A los EP se les pedía analizar dichos problemas identificando los conceptos y procedimientos matemáticos 
implicados en la resolución de cada uno de ellos, y formular preguntas (cuatro para cada problema) que podrían plantear en una situación de enseñanza, indicando lo que se pretendía con cada una de ellas. En este caso, las herramientas conceptuales procedían de la información proporcionada en la parte teórica de la asignatura y de artículos de revistas suministrados en la parte práctica (Figueiras, 2013).

La elección de estas tareas fue condicionada por dos razones: por un lado, abarcaban actividades profesionales, como analizar, interpretar y formular preguntas, las cuales están muy presentes en la labor de los maestros; por otro lado, estaban situadas al final del curso académico, con lo que los subgrupos de trabajo estaban más cohesionados y familiarizados con la forma de trabajar.

\section{Recolección de datos}

La fuente de datos de este estudio fueron las transcripciones de las grabaciones en audio de los diálogos verbales de los EP mantenidos mientras resolvían las dos tareas didáctico-matemáticas propuestas en el aula. Concretamente, cada una de las tareas fue implementada durante cinco sesiones de una hora semanal, a lo largo de las cuales procedimos a la recolección de estas grabaciones en audio.

Con objeto de facilitar la gestión de la información obtenida, las transcripciones fueron analizadas con el programa informático Atlas.ti para identificar narrativas, que pasaron a ser los datos del estudio que aquí presentamos.

\section{Análisis de datos}

De todas las narrativas identificadas con Atlas.ti se seleccionaron aquellas que eran endosadas dentro del subgrupo y asumidas por todos sus miembros. Posteriormente, las narrativas endosadas que compartían una misma idea relacionada con uno o varios elementos de la triada fueron agrupadas bajo diferentes códigos. Así, narrativas relacionadas con elementos de la triada y que compartían la misma idea de la importancia de tener en cuenta las respuestas de los niños fueron agrupadas bajo el código TP-PRN (tarea profesional-pensar en cómo responderán los niños).

A continuación, se seleccionaron los grupos de narrativas que tenían un carácter prescriptivo, o sea, aquellas que de alguna manera condicionaban la forma de actuación de estos EP. El estudio de estos grupos de narrativas nos llevó a inferir el significado subyacente que parecían expresar, identificando este significado como una norma y expresándolo con nuestras propias palabras. Es decir, las normas se infieren y etiquetan por el investigador a partir de repetidas narrativas expresadas por los participantes en el estudio. 
En la tabla 1 presentamos un ejemplo de la forma en la que hemos inferido la norma.

Tabla 1

Ejemplo del proceso seguido para inferir las normas

Ejemplos de narrativas endosadas

SG3 [Contexto: formulando una pregunta para uno de los problemas relacionados con la probabilidad]

715: EP6: ¿Qué pasaría si, en vez de tirar un dado, tiraran dos? Es qué pasaría, pero a lo mejor pueden pensar..

716: EP4: ¿Que qué pasaría si tiraran dos?

717: EP6: Pues que uno va a tener 6 posibilidades y el otro 2, o sea, si...

718: EP3: Es que en realidad de un ejercicio que tiene unos objetivos, sacar 4 preguntas con distintas finalidades de cada pregunta...

719: $\quad$ EP6: $\quad$ Es que a lo mejor este puede estar bien para que, para pretender, en este caso para que vean que la cantidad no es importante a la hora de tener más posibilidad o no de sacar ese número, ¿sabes?

720: EP3: Eso sí.

721: EP6: Porque a lo mejor pueden entender que, teniendo dos dados ahora, Abel pueda estar posicionado de la misma manera que Rosa.

722: EP4: Vale.

723: EP3: También pueden pensar que si Abel tiene un 5, también es más que un 3, un 2 o un 1, ¿sabes?

724: EP4: Claro.
Las preguntas planteadas pueden permitir diferentes respuestas/ pensamientos en los niños. TP-PRN

"En las tareas profesionales es importante tener en cuenta las posibles respuestas de los niños".

SG5 [Contexto: identificando los procedimientos implicados en uno de los problemas relacionados con la probabilidad]

22: EP4: Y los procedimientos serían como el niño llega a la conclusión final, ¿no?

23: EP1: Sí, para resolver esa tarea.

24: EP4: El procedimiento a lo mejor podría ser poner en plan que el niño se haga un dibujo y que vea, o sea que coloree las bolas de los colores, ¿no?

Identificar los procedimientos a través de las posibles resoluciones de los niños.

TP-PRN 
SG4 [Contexto: analizando la actividad escolar que aparece en la primera tarea]

94: EP4: No entiendo yo, si tú le dices a alguien "Reconoce los cuadriláteros que hay aquí", pues que el niño te diga los cuadriláteros es que tienen cuatro lados, otro pues lo que tiene los vértices.

95: EP1: Yo creo que el niño también, porque pone aquí análisis, el niño está sobreavisado de propiedades de una figura, yo pienso que sí porque, por ejemplo, el niño sabe cuadrado que tiene cuatro lados y no sé qué, no sé cuánto, entonces el niño lo tiene que saber para reconocer que eso es un cuadrado, yo creo que esa estrategia sí la usa el niño, ¿no? Bueno, es que eso en verdad es estrategia visual.

96: EP2: Yo creo que depende, porque él [refiriéndose al niño] igual reconoce un cuadrado como aquello que se parece a un dado y todo lo que vea que tiene forma de dado es un cuadrado para ellos [refiriéndose a los niños], por ejemplo.

SG8 [Contexto: analizando una pregunta de la actividad escolar que aparece en la primera tarea]

2: EP2: [Leyendo una de las preguntas de la actividad escolar incluida en la primera tarea] ¿En qué le dirías a alguien que se fijara para localizar todos los rectángulos en una lámina de figuras?

3: $\quad$ EP3: Pero esto hay que pasárselo a los niños.

4: EP2: Sí, yo creo que sería, yo creo que un niño diría que se fijase... es que... ¿en qué curso están?, ¿en segundo ciclo?

5: EP4: Pero saben que...

6: EP1: Que tengan dos lados iguales, dos a dos y más delgado que el cuadrado.

7: $\quad$ EP2: Yo creo que si supieran lo que es el ángulo recto, dirían que es un polígono o una figura que tiene cuatro ángulos rectos y que tiene los lados paralelos dos a dos, eso es segundo ciclo de primaria y ya está, ¿no?

8: $\quad$ EP1: $\quad$ Tercero y cuarto de primaria.

9: EP2: Eso es 8 o 9 años.

10: EP1: En los libros de primaria te viene paralelos dos a dos.

11: EP2: Claro.
Considerar las posibles respuestas de los niños para analizar la actividad escolar.

TP-PRN

"En las tareas profesionales es importante tener en cuenta las posibles respuestas de los niños".
Considerar las posibles respuestas de los niños para analizar las cuestiones planteadas en la actividad escolar.

TP-PRN

Fuente: elaboración propia

Finalmente, siguiendo nuestro marco teórico, las normas inferidas fueron analizadas para aproximarnos a su naturaleza social, sociomatemática, socio-didáctico-matemática o didáctico-matemática, discutiendo los matices que podrían llevar a una u otra consideración. 


\section{Resultados}

Los resultados que aquí presentamos corresponden, como indicamos inicialmente, a 11 subgrupos (45 EP) con un mismo profesor y con dos tareas didáctico-matemáticas.

Tanto en el "discurso como alumnos" como en el "discurso como futuros profesores" se han podido inferir diferentes normas, que pasamos a describir a continuación.

\section{Normas inferidas en el discurso como alumnos}

Los EP traen unas normas previas que se identifican en el discurso que generan al resolver tareas didáctico-matemáticas. Estas normas, provenientes de sus experiencias escolares anteriores, pueden estar vinculadas a la forma de considerar la actividad que se desarrolla en cualquier aula en un contexto escolar (consideradas normas sociales) o más vinculadas a las clases de matemáticas (consideradas normas sociomatemáticas). Particularizando en este caso en un profesor universitario en una clase de didáctica de las matemáticas, estas normas se pueden considerar socio-didácticomatemáticas. En nuestro estudio las normas que hemos inferido se recogen en la tabla 2, que se muestra a continuación:

Tabla 2

Normas inferidas en el discurso como alumnos

\begin{tabular}{|c|c|c|}
\hline \multicolumn{2}{|r|}{ Normas inferidas } & \multirow{2}{*}{$\begin{array}{l}\text { Subgrupos de EP en los que se han } \\
\text { identificado las categorías de } \\
\text { narrativas que documentan la norma } \\
\text { SG1, SG3, SG4, SG5, SG6, SG7, SG9, } \\
\text { SG10 y SG11 }\end{array}$} \\
\hline N1 & $\begin{array}{l}\text { "Aunque se tenga la información necesaria, es el } \\
\text { profesor en el aula el que tiene que proporcionar el } \\
\text { contenido que se necesita para hacer la tarea". }\end{array}$ & \\
\hline N2 & $\begin{array}{l}\text { "Es necesario saber lo que el profesor espera que } \\
\text { hagamos en la tarea". }\end{array}$ & $\begin{array}{l}\text { SG1, SG3, SG4, SG5, SG6, SG7, SG9, } \\
\text { SG10 y SG11 }\end{array}$ \\
\hline N3 & "Lo que dice el profesor no se discute". & SG4, SG5, SG6, SG9 y SG11 \\
\hline N4 & $\begin{array}{l}\text { "El profesor tiene que confirmar los resultados } \\
\text { y validar lo hecho". }\end{array}$ & $\begin{array}{l}\text { SG1, SG3, SG4, SG5, SG6, SG7, SG9, } \\
\text { SG10 y SG11 }\end{array}$ \\
\hline N5 & "Cuánto más se ponga en la respuesta mejor". & SG2, SG3, SG5, SG6, SG7 y SG10 \\
\hline N6 & $\begin{array}{l}\text { "Hay que responder todas las preguntas que } \\
\text { se plantean en la tarea". }\end{array}$ & $\begin{array}{l}\text { SG2, SG3, SG4, SG5, SG6, SG7, } \\
\text { SG9 y SG10 }\end{array}$ \\
\hline N7 & $\begin{array}{l}\text { "Analizar e interpretar es aplicar directamente un } \\
\text { contenido didáctico-matemático". }\end{array}$ & $\begin{array}{l}\text { SG1, SG2, SG3, SG4, SG5, SG6, SG7, } \\
\text { SG8, SG9, SG10 y SG11 }\end{array}$ \\
\hline N8 & $\begin{array}{l}\text { "Analizar tareas es hacer exclusivamente lo que se pide } \\
\text { que hagan los niños a los que van dirigidas las mismas". }\end{array}$ & SG2, SG5, SG6, SG7, SG8, SG9 y SG10 \\
\hline N9 & $\begin{array}{l}\text { "Analizar tareas de probabilidad, en las que se pide } \\
\text { identificar los conceptos y procedimientos que } \\
\text { demandan, es buscarlos en las palabras que aparecen } \\
\text { en el enunciado, sin ninguna reflexión más". }\end{array}$ & SG3, SG5, SG6 y SG10 \\
\hline
\end{tabular}


Como se puede ver en esta tabla, las cuatro primeras normas hacen referencia a la forma de actuar del estudiante en el aula con respecto al profesor y las cinco restantes están relacionadas con la forma de actuar del estudiante con las tareas.

Con respecto a las primeras, tres aparecen en casi todos los subgrupos (N1, N2 y N4). La N1 muestra la importancia que tiene para estos EP la explicación previa del profesor sobre los contenidos que aparecen en la tarea, porque ellos entienden que la necesitan para poder realizarla. A pesar de tener otras fuentes de información a las que poder recurrir, como son en este caso las lecturas, los subgrupos ven al profesor universitario como la única fuente de información. A diferencia de esta norma, en la que los EP se centran en los contenidos, en la N2 el foco está puesto en cumplir las expectativas que el profesor tiene sobre la tarea. Para estos EP es imprescindible que el profesor indique lo que hay que hacer exactamente en la tarea para poder continuar con su realización. Sin esta información, ellos tienen dificultades o reticencias para continuar. En algunas de las narrativas endosadas incluidas en esta norma se aprecia que las preguntas van más encaminadas a tratar de "adivinar lo que quiere el profesor que se haga" que a querer saber lo que hay que hacer en la tarea.

La norma N3 corresponde a narrativas endosadas en las que se aprecia cómo algo, por el mero hecho de decirlo el profesor, es aceptado por todo el subgrupo, sin llegar a cuestionarlo ni ser discutido, a pesar de que algunos componentes del subgrupo a veces pensaran de manera diferente antes de preguntar al profesor. Cualquier comentario del profesor lleva automáticamente a su aceptación por todos los miembros del subgrupo sin más discusión.

En las narrativas endosadas que han llevado a inferir la norma N4 se aprecia cómo, para estos EP, aspectos tales como confirmar lo que está hecho, validar las respuestas dadas, clarificar dudas en las respuestas, etc., caracterizan el papel del profesor en todo momento (en su pasado, su presente $y$, probablemente, en su futuro profesional).

Por otro lado, las normas N5, N6, N7, N8 y N9 están relacionadas con las tareas. Concretamente, las dos primeras se pueden dar en cualquier tipo de tareas y las otras se refieren a tareas profesionales del profesor.

La N5 expresa una idea vinculada a que cuanto más se escriba, como respuesta a una tarea planteada por el profesor, mejor. Para los EP, cuanta más información pongan en la respuesta, aunque esta información sea redundante o irrelevante, mejor respondida está la tarea. En este caso llegan a incluir información proporcionada por el profesor, por las lecturas y por los propios miembros del subgrupo, lo que da lugar, en muchas ocasiones, a una redundancia en la respuesta. Relacionada con esta norma, pero con un matiz diferente, la N6 refleja la idea de que no es correcto dejar 
sin rellenar los apartados de la tarea, lo que lleva, en ocasiones, a recoger comentarios repetidos o a forzar la inclusión de contenidos poco relacionados. Seis de los subgrupos muestran las dos últimas normas, N5 y N6, lo que puede indicar una cierta relación entre ellas.

La prevalencia de la norma N7, ya que aparece en todos los subgrupos, puede indicar que las tareas didáctico-matemáticas siguen siendo consideradas por los estudiantes como "tareas de aplicación", consideración que puede deberse a la gran frecuencia con que se han enfrentado a tareas de ese tipo en las aulas de matemáticas en los niveles anteriores y el fuerte arraigo generado.

En las narrativas endosadas que nos han llevado a inferir la norma N8, se aprecia cómo estos EP resuelven la actividad escolar que tienen que analizar sin hacer ningún tipo de reflexión posterior a la resolución. Es decir, se sitúan como alumnos de educación primaria que tienen que resolver la actividad escolar y no como profesores, ya que cuando terminan de resolverla dan por concluida la tarea profesional de analizar.

La última norma, N9, está vinculada con un análisis de los conceptos y procedimientos involucrados en una actividad escolar. Para ello, los EP buscan palabras que aparecen en el enunciado de la actividad escolar, pues las entienden como los conceptos y procedimientos pedidos.

Algunas de estas normas han sido ya identificadas en otros trabajos (Sánchez \& García, 2014; Toscano et al., 2013), lo que nos ha permitido contrastarlas y apreciar su persistencia. Otras, aunque pueden formar parte de declaraciones evidentes de antemano, en el sentido de ser compartidas por gran parte del colectivo de profesores, han sido identificadas en este trabajo como normas, por su carácter prescriptivo para estos EP.

A continuación, pasamos a aproximarnos a la naturaleza que presentan estas normas identificadas. En este estudio, hemos optado por una presentación descriptiva textual de la naturaleza de estas normas y no por un formato de tabla, por el carácter interpretativo de dicha naturaleza. Entre las normas aquí identificadas, algunas de ellas, en determinados contextos y situaciones, podrían considerarse de otro tipo.

De las cuatro primeras se puede decir que tienen una naturaleza social, ya que han sido establecidas por unos profesores genéricos y por ellos como estudiantes en clases anteriores, de modo que han configurado un rol de profesor y de estudiantes independientemente del contenido dado. Estas normas se pueden considerar como previas y trasladadas directamente por estos EP al contexto de un aula universitaria de didáctica de las matemáticas. De las otras cinco normas relacionadas con las tareas, por un lado, dos de ellas presentan una visión de las tareas generadas en clases escolares anteriores (N5 y N6), las cuales también se trasladan directamente a las tareas didáctico-matemáticas consideradas como una tarea a 
cumplimentar. Estas normas tendrían una naturaleza social. Por otro lado, las tres restantes, aunque estén relacionadas con las tareas profesionales, muestran la traslación que se hace de normas sociomatemáticas en las que la aplicación de un contenido, la búsqueda de un resultado a todo problema planteado y la búsqueda de palabras clave pasan a tenerse en cuenta en la resolución de las tareas profesionales, con lo que se considera de naturaleza socio-didáctico-matemática.

En conjunto, todas las normas inferidas en el discurso como alumnos son, por sus características, muy próximas a un papel del profesor y a una visión de las tareas generada en clases previas, en general, y de matemáticas, en particular, que el EP traslada a las aulas de didáctica de las matemáticas. Son, por tanto, unas normas sociales o sociomatemáticas que se mantienen o se adaptan como normas socio-didáctico-matemáticas cuando los EP se sitúan en el discurso como alumnos universitarios que resuelven una tarea profesional.

\section{Normas inferidas en el discurso como futuros profesores}

En menor medida, los EP se situaron en un discurso como futuros profesores, a pesar de que la situación planteada en la tarea didáctico-matemática de alguna manera trataba de situarles en este rol. En este discurso, las normas inferidas adquieren un carácter socio-didáctico-matemático, al estar centradas en la forma de considerar la enseñanza y el aprendizaje de las matemáticas escolares como una materia propia de su formación específica. También se pueden apreciar normas didáctico-matemáticas relacionadas con contenidos propios de Didáctica de las Matemáticas. Entre las normas en este discurso, con respecto al papel que ellos piensan que debería tener un profesor de educación primaria, se han inferido las normas que se recogen en la tabla 3.

Tabla 3

Normas inferidas en el discurso como futuros profesores

\begin{tabular}{|c|c|c|}
\hline \multicolumn{2}{|r|}{ Normas inferidas } & $\begin{array}{l}\text { Subgrupos de EP en los que se han } \\
\text { identificado las categorías de } \\
\text { narrativas que documentan la norma } \\
\text { SG6 y SG7 }\end{array}$ \\
\hline N11 & $\begin{array}{l}\text { "En las tareas profesionales es importante tener en cuenta } \\
\text { las posibles respuestas de los niños". }\end{array}$ & SG3, SG4, SG5, SG8 y SG9 \\
\hline N13 & $\begin{array}{l}\text { "Para analizar tareas, hay que hacer lo que se pide a los } \\
\text { niños como punto de partida para el análisis posterior". }\end{array}$ & SG1, SG3, SG5, SG6, SG7, SG9, SG10 y SG11 \\
\hline N14 & $\begin{array}{l}\text { "Hay que plantear preguntas encaminadas a favorecer la } \\
\text { comprensión de un concepto". }\end{array}$ & SG3, SG9 y SG10 \\
\hline
\end{tabular}

Fuente: elaboración propia 
En las narrativas endosadas que nos han llevado a inferir la norma N10, se aprecia cómo los EP consideran que como profesores tienen que proporcionar el contenido implicado en una actividad escolar para que los alumnos puedan realizarla.

Queremos resaltar que la N1 y la N10 están muy relacionadas, pero con matices diferentes. En la N1, los EP se sitúan como alumnos que necesitan que el profesor universitario les proporcione el contenido, y en la N10 los EP adoptan el papel de profesores al sentir la necesidad de proporcionarlo. Esto nos informa de la transición de un rol como alumnos a uno como profesores, pero, sin embargo, se ha traslado lo que ocurría en las aulas.

Por otro lado, las normas N11, N12, N13, N14 y N15 están más relacionadas con las tareas profesionales de un profesor.

En la N11 se recoge cómo los EP consideran posibles respuestas de alumnos a la hora de abordar las tareas profesionales. Por ejemplo, ante la tarea profesional de analizar actividades escolares teniendo en cuenta el razonamiento geométrico que puede estar implicado en su resolución, los EP tratan de aportar posibles respuestas de los alumnos a la actividad escolar para ver qué distintos niveles de razonamiento geométrico podría demandar la actividad escolar. De algún modo empieza a apreciarse que estos EP consideran la capacidad de predecir respuestas de los alumnos como algo necesario para realizar su labor como futuros profesores.

En las narrativas endosadas que nos han permitido inferir la norma N12 se aprecia cómo estos EP empiezan a tener en cuenta el contenido didáctico-matemático como un conocimiento específico que debe poseer un profesor para abordar las tareas profesionales. A diferencia de lo que sucedía en el discurso como alumnos, este reconocimiento no es una simple aplicación del contenido didáctico-matemático, sino una búsqueda en ese contenido de elementos que permitan resolver la tarea profesional que están abordando.

Por último, las tres últimas normas (N13, N14 y N15) están vinculadas a tareas profesionales concretas. La N13 está relacionada con el análisis de actividades escolares, y los EP consideran necesario realizar la actividad escolar como un primer paso para hacer una interpretación posterior de ella. En cierto modo, esta norma marca una transición entre su papel como alumno, que resuelve exclusivamente la actividad escolar (véase la N8), y su futura actividad profesional. Las otras dos normas (N14 y N15) están relacionadas con el planteamiento de preguntas en el aula por parte del profesor. La N14 se infiere de narrativas en las que los EP consideran que plantear preguntas es un medio de favorecer el aprendizaje de un concepto por parte de los alumnos. En otras narrativas se aprecia que estos EP consideran que las preguntas que 
tienen que plantear son una guía para facilitar la resolución de la actividad escolar a los alumnos.

Con respecto a la naturaleza de las normas identificadas en este discurso como profesores, la primera de las normas (N10) muestra una traslación del rol del profesor, conformado en los niveles educativos previos en los que ellos han sido los estudiantes, a su futuro rol como profesores, por lo que se podría considerar su carácter social. Del mismo modo, la norma N15 también muestra un paso de una norma sociomatemática, en este caso, en la que se buscan palabras clave en el enunciado de los problemas para resolverlos, a una socio-didáctico-matemática, en la que se ve necesario que el profesor formule preguntas para facilitar esa búsqueda. Las otras cuatro normas (N11, N12, N13 y N14) podrían considerarse como la emergencia de normas específicas propias en el aula de Didáctica de las Matemáticas. La capacidad de predecir respuestas de los niños, la valoración de los contenidos didáctico-matemáticos, la importancia de reflexionar sobre lo que demandan las actividades escolares y la importancia dada a la comprensión de conceptos marcan una transición entre su papel como alumnos que resuelven una tarea y su futuro papel como profesores ante una actividad profesional. El que estas normas sean consideradas didáctico-matemáticas o socio-didáctico-matemáticas depende de la medida en que sean asumidas por la comunidad científica de didáctica de las matemáticas o sean más específicas de la propia aula en la que se han identificado.

Se puede decir que estas normas nos permiten aproximarnos a una etapa inicial de su desarrollo como (futuros) profesores. Es importante destacar que algunas de estas normas indican que el papel del profesor sigue trasladando una interacción establecida entre el profesor y los alumnos que está centrada en el profesor. Sin embargo, la relación de los EP y las tareas didáctico-matemáticas indica de algún modo el uso del contenido didáctico-matemático y la importancia de tener en cuenta a los alumnos, lo que serían unos aspectos importantes en su futura labor profesional. Llama la atención los pocos subgrupos en los que se han inferido estas normas en este tipo de discurso, a diferencia de lo que sucedía en el apartado anterior. Esto podría deberse a la dificultad que tienen en asumir un papel de profesor, a pesar de las demandas de la tarea planteada.

Finalmente, si tenemos en cuenta las normas inferidas en los dos discursos, podemos apreciar que en ambos se han identificado normas de naturaleza socio-didáctico-matemática, pero en cada uno de los discursos presentan un matiz diferente. En el discurso como alumnos, a las normas se les da el carácter socio-didáctico-matemático porque son normas previas (ya sean sociales o sociomatemáticas) que se trasladan a un contenido didáctico-matemático. Sin embargo, en el discurso como futuros profesores, 
las normas socio-didáctico-matemáticas emergen del propio contenido didáctico-matemático.

\section{Discusión y conclusiones}

Nuestro propósito en este artículo ha sido indagar sobre las normas que regulan el discurso de unos estudiantes en un contexto de formación de profesores de primaria. Sin ánimo en absoluto de generalizar, se puede decir que el papel adoptado por estos EP en la resolución de unas tareas didáctico-matemáticas ha generado diferentes discursos reglados por diferentes normas, lo que nos informa sobre el primer objetivo de este estudio.

Como se ha mostrado en el estudio, los futuros profesores participantes en él, cuando adoptan el papel de alumnos universitarios, trasladan a las aulas unas normas sociales y socio-matemáticas provenientes de experiencias anteriores, que regulan su rol como alumnos y afectan al desarrollo de las tareas didáctico-matemáticas planteadas, lo que nos permite valorar el papel que pueden desempeñar. El que algunas de estas normas hayan sido identificadas en diferentes contextos y etapas educativas (Sánchez \& García, 2014; Yackel \& Cobb, 1996) conduce, por un lado, a la necesidad de realizar un estudio profundo de lo que sucede en otros niveles, valorando otros aspectos, como pueden ser el sociohistórico y el escolar (Martin, 2000). Y, por otro lado, hace que se preste una especial importancia a la transición entre la escuela/instituto y la universidad, sobre todo en un contexto de formación de profesores. Más información sobre si las normas identificadas en el discurso como alumnos de estos EP son propias de una determinada cultura escolar o transversales a diferentes culturas podría ayudar a entender los diferentes resultados obtenidos en la implementación de programas docentes o en investigaciones llevadas a cabo en ellos.

Cuando el papel adoptado genera un discurso más próximo al de un profesor, se infieren de este discurso unas normas que muestran la generación de una incipiente gestación de su papel como profesores. El que se hayan inferido en este caso menos normas y en un número menor de subgrupos puede deberse a la dificultad que tienen al enfrentarse al tipo de tareas planteadas o a que estos EP se han ido adoctrinando en las normas escolares mediante la continua participación en el rol de estudiantes, pero, sin embargo, no se han adoctrinado con las normas relativas al rol del profesor, ya que no han asumido ese papel (Holland, Lachicotte, Skinner \& Cain, 1998), lo que vuelve a indicar la importancia de las prácticas docentes vinculadas con los programas de formación. Algunas de estas normas llevan aparejada una continuidad de las nomas identificadas como alumnos, 
lo que puede llevar a mantener en ese incipiente papel una interacción en el aula centrada en el profesor, dando poca autonomía a sus futuros alumnos. Por otro lado, la emergencia de rasgos como la importancia dada a la capacidad de predecir las respuestas de los alumnos como algo a tener en cuenta en el desarrollo de las tareas profesionales aquí planteadas (interpretar, analizar, etc.) emerge como una competencia para la práctica docente que ayuda a ser consciente de la diversidad que se da en las aulas escolares.

Los resultados obtenidos tienen una clara repercusión en relación con la formación de profesores, ya que ayudan a reconocer otros aspectos, como las normas identificadas en este estudio, que pueden emerger como obstáculos en esa formación y que pueden minimizar el papel de las reformas curriculares o metodológicas con las que se intenta mejorarlas. La toma de conciencia del papel que pueden cumplir las normas, junto con la inmersión en comunidades de práctica (Wenger, 1998), puede ayudar a generar un discurso didáctico-matemático en los EP que permita abordar las tareas que se desarrollan en los programas de formación desde una perspectiva profesional.

Queremos señalar que este estudio no ha sido en absoluto un "estudio de déficit", en el que se trate de poner de manifiesto normas que puedan afectar negativamente el futuro desarrollo profesional y se busquen responsabilidades en otros niveles educativos. Lo que aquí hemos intentado es aproximarnos a las diferentes normas que pueden subyacer en la forma de considerar la enseñanza/aprendizaje de unos estudiantes universitarios, futuros profesores de primaria, que han participado en este estudio, desde una doble perspectiva. En primer lugar, buscando su posible procedencia, con base en las normas sociales y sociomatemáticas identificadas en estudios previos, que nos pueden informar del carácter y la persistencia de estas, o de su especificidad, muy vinculadas con las tareas planteadas en este estudio. Y en segundo lugar, la identificación en este caso concreto de algunas normas socio-didáctico-matemáticas y didáctico-matemáticas que pueden ser indicadoras de su consideración del papel del profesor y su posible actuación en su futura labor profesional. Una profundización en estos aspectos puede ser objetivo de futuras investigaciones.

Finalmente, dado el carácter exploratorio de nuestro estudio, hemos intentado aproximarnos a lo que sucede en un contexto muy concreto, con unas tareas y unos EP. Pensamos que puede ser un primer punto de partida para ampliar el estudio a otras tareas-colectivos, intentando visibilizar el papel de las normas que pueden trasladarse a la formación de profesores y afectar los procesos de enseñanza/aprendizaje de las matemáticas de sus futuros alumnos. 


\section{Sobre los autores}

Rocío Toscano es licenciada en Matemáticas y estudiante del Doctorado en Educación en la Universidad de Sevilla, España. Es profesora en el Departamento de Didáctica de las Matemáticas de la Universidad de Sevilla, España. Su investigación se centra en la formación de profesores de matemáticas de distintos niveles educativos.

José María Gavilán-Izquierdo es licenciado en Matemáticas, doctor en Didáctica de las Matemáticas y profesor titular de la Universidad de Sevilla. Es investigador responsable del grupo de investigación en Educación Matemática (FQM226). Su investigación se centra en niveles universitarios.

Victoria Sánchez es catedrática jubilada de la Universidad de Sevilla. Actualmente es colaboradora honoraria en el Departamento de Didáctica de las Matemáticas de la Universidad de Sevilla, España. Su trayectoria investigadora ha estado vinculada con la formación inicial de profesores en relación con las matemáticas.

\section{Referencias}

Biddle, B. J. \& Thomas, E. J. (1996). Prescriptions. En B. J. Biddle \& E. J. Thomas (Eds.), Role Theory: Concepts and Research (pp. 103-104). Nueva York: Wiley and Sons.

Burger, W. F. \& Shaughnessy, J. M. (1986). Characterizing the Van Hiele levels of development in Geometry. Journal for Research in Mathematics Education, 17(1), 31-48.

Caspi, S. \& Sfard, A. (2012). Spontaneous meta-arithmetic as a first step toward school algebra. International Journal of Educational Research, 51-52, 45-65. doi: 10.1016/j.ijer.2011.12.006

Figueiras, L. (2013). ¿Por qué no empezar a formular buenas preguntas? Cuadernos de Pedagogía, 438(1), 55-59. Recuperado de https://ddd.uab.cat/pub/ artpub/2013/163908/cuaped_a2013m10n438p55.pdf

Gee, J. P. (1996). Social Linguistics and Literacies: Ideology in Discourses. Nueva York: Routledge Falmer.

Gee, J. P. (2001a). Reading as situated language: A sociocognitive perspective. Journal of Adolescent \& Adult Literacy, 44(8), 714-725. Recuperado de https://www.jstor.org/stable/40018744

Gee, J. P. (2001b). Identity as an analytic lens for research in education. En W. G. Secada (Ed.), Review of Research in Education (pp. 99-125). Washington: American Educational Research Association.

Gorgorió, N. \& Planas, N. (2005). Social representations as mediators of mathematical learning in multiethnic classrooms. European Journal of Psychology of Education, $X X(1), 91-104$. doi: 10.1007/BF03173213

Gutiérrez, A. \& Jaime, A. (1998). On the assessment of the Van Hiele levels of reasoning. Focus on Learning Problems in Mathematics, 20(2-3), 27-46. 
Herbel-Eisenmann, B. (2003). Examining "Norms" in Mathematics Education Literature: Refining the Lens. Recuperado de https://eclass.uowm.gr/modules/ document/file.php/ELED257/EPГA $\Sigma$ IA\%3A\%20ANA^Y $\Sigma H \% 20 E \prod E I \Sigma O \triangle I$

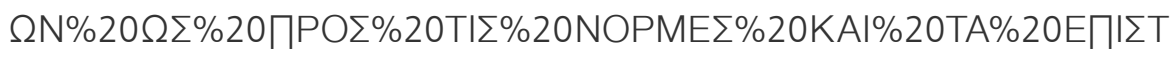

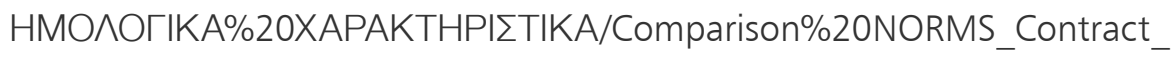
NCTM2003.pdf

Hoffer, A. (1981). Geometry is more than proof. Mathematics Teacher, 74(1), 11-18.

Holland, D., Lachicotte, W. Jr., Skinner, D. \& Cain, C. (1998). Identity and Agency in Cultural World. Cambridge: Harvard University Press.

Hunter, R. K. \& Anthony, G. (2011). Forging mathematical relationships in inquiry-based classrooms with Pasifika students. Journal of Urban Mathematics Education, 4(1), 98-119. Recuperado de https://pdfs.semanticscholar. org/5feb/d56ac5ab7a7e7a7114b0152e753c591a0658.pdf

Jaime, A. \& Gutiérrez, A. (1990). Una propuesta de fundamentación para la enseñanza de la geometría: el modelo de Van Hiele. En S. Llinares \& V. Sánchez (Eds.), Teoría y práctica en Educación Matemática (pp. 295-384). Sevilla: Alfar.

Lave, J. \& Wenger, E. (1991). Situated Learning: Legitimate Peripheral Participation. Cambridge: Cambridge University Press.

Martin, D. B. (2000). Mathematics Success and Failure among African-American Youth: The Roles of Sociohistorical Context, Community Forces, School Influence, and Individual Agency. Mahwah: Lawrence Erlbaum Associates.

Mercer, N. (2004). Sociocultural discourse analysis: Analysing classroom talk as a social mode of thinking. Journal of Applied Linguistics, 1(2), 137-168. doi: 10.1558/japl.v1i2.137

Moschkovich, J. (2007). Examining mathematical discourse practices. For the Learning of Mathematics, 27(1), 24-30. Recuperado de https://www.jstor. org/stable/40248556

Sánchez, V. \& García, M. (2009). Tasks for primary student teachers: A task of mathematics teacher educators. En B. Clarke, B. Grevholm \& R. Millman (Eds.), Tasks in Primary Mathematics Teacher Education. Purpose, Use and Exemplars (pp. 37-50). Dordrecht: Springer.

Sánchez, V. \& García, M. (2011). Socio-mathematical and mathematical norms in pre-service primary teachers' discourse. En B. Ubuz (Ed.), Proceedings of the $35^{\text {th }}$ Conference of the International Group for the Psychology of Mathematics Education (pp. 105-112). Turquía: PME.

Sánchez, V. \& García, M. (2014). Sociomathematical and mathematical norms related to definition in pre-service primary teachers' discourse. Educational Studies in Mathematics, 85(2), 305-320. doi: 10.1007/s10649-013-9516-0

Sfard, A. (2001). There is more to discourse than meets the ears: Looking at thinking as communicating to learn more about mathematical learning. Educational Studies in Mathematics, 46(1-3), 13-57. doi: 10.1023/A:1014097416157

Sfard, A. (2006). Participacionist discourse on mathematics learning. En J. Maasz \& W. Schlöglmann (Eds.), New Mathematics Education Research and Practice (pp. 153-170). Rotterdam: Sense Publishers B. V. 
Sfard, A. (2007). When the rules of discourse change, but nobody tells you: Making sense of mathematics learning from a commognitive standpoint. The Journal of the Learning Sciences, 16(4), 567-615. doi: 10.1080/10508400701525253

Sfard, A. (2008). Thinking as Communicating: Human Development, the Growth of Discourses and Mathematizing. Cambridge: Cambridge University Press.

Tatsis, K. \& Koleza, E. (2008). Social and socio-mathematical norms in collaborative problem-solving. European Journal of Teacher Education, 31(1), 89-100. doi: 10.1080/02619760701845057

Toscano, R., Gavilán-Izquierdo, J. M. \& Sánchez, V. (2019). A study of pre-service primary teachers' discourse when solving didactic-mathematical tasks. Eurasia Journal of Mathematics, Science and Technology Education, 15(11), em1762. doi: 10.29333/ejmste/108631

Toscano, R., Sánchez, V. \& García, M. (2013). Norms and perspectives in pre-service secondary mathematics teachers' discourse. En A. M. Lindmeier \& A. Heinze (Eds.), Proceedings of the 37th Conference of the International Group for the Psychology of Mathematics Education (pp. 289-296). Kiel: PME.

Vygotsky, L. S. (1978). Interaction between learning and development. En M. Cole, V. John-Steiner, S. Scribner \& E. Souberman (Eds.), Mind in Society: Development of Higher Psychological Processes (pp. 79-91). Cambridge: Harvard University Press.

Wells, G. \& Claxton, G. (Eds.). (2002). Learning for Life in the 21st Century: Sociocultural Perspectives on the Future of Education. Oxford: Blackwell.

Wenger, E. (1998). Communities of Practice: Learning, Meaning and Identity. Nueva York: Cambridge University Press.

Wertsch, J. V. (1985). Culture, Communication and Cognition: Vygotskian Perspectives. Cambridge: Cambridge University Press.

Yackel, E. \& Cobb, P. (1996). Sociomathematical norms, argumentation, and autonomy in mathematics. Journal for Research in Mathematics Education, 27(4), 458-477. doi: 10.2307/749877 